\title{
Avaliação do Impacto dos Programas de Reorientação Profissional em Cursos da Área da Saúde
}

\section{Evaluation of the Impact of Professional Reorientation Programs in Health Courses}

\author{
Bibiana Arantes Moraes ${ }^{I}$ \\ Lucilene Maria de Sousal \\ Ida Helena Carvalho Francescantonio Menezes ${ }^{I}$ \\ Maria Goretti Queiroz \\ Nilce Maria da Silva Campos Costa \\ Marília Mendonça Guimarães ${ }^{\perp}$ \\ Beatriz Jansen FerreiraII
}

\section{PALAVRAS-CHAVE}

- Avaliação.

- Pró-Saúde.

- PET-Saúde.

- Reestruturação Curricular.
I Universidade Federal de Goiás, Goiânia, Goiás, Brasil.

${ }^{\text {II } U n i v e r s i d a d e ~ E s t a d u a l ~ d e ~ C a m p i n a s . ~}$
Problematizou-se a percepção dos atores dos cursos da área da saúde da Universidade Federal de Goiás quanto ao impacto do Programa Nacional de Reorientação da Formação Profissional em Saúde articulado ao Programa de Educação pelo Trabalho para a Saúde (Propet-Saúde) na reestruturação curricular. Realizou-se um estudo transversal com a participação de 300 atores de sete cursos, entre estudantes, tutores, preceptores, professores e coordenadores de graduação. Para isto, foi construído e validado um instrumento atitudinal do tipo Likert com duas dimensões: D1: reestruturação curricular e os princípios do Propet-Saúde e D2: projeto pedagógico do curso e o Propet-Saúde. A validação de conteúdo do instrumento considerou as equivalências semântica, cultural e conceitual, atribuição de pontos (de 1 a 4) e randomização das assertivas. A pontuação gerou três intervalos interpretativos: de 1 a 1,99 (insatisfação); 2 a 2,99 (relativa satisfação) e 3 a 4 (satisfação). Foi feito pré-teste e, após a inserção das contribuições, o instrumento foi aplicado. Após a aplicação, o instrumento foi validado estatisticamente quanto à dispersão e confiabilidade. Foi realizada análise fatorial considerando função e curso. A dispersão por meio da correlação linear evidenciou perda de quatro asserções $(11,77 \%)$ denotando excelente validação de conteúdo; a confiabilidade foi de 0,93 ( $p<0,01)$, evidenciando ótima densidade estatística. As médias gerais de D1 e D2 foram 3,09 e 3,11 respectivamente, indicando satisfação entre os respondentes. Conclui-se que os participantes reconhecem importantes avanços, como a reestruturação curricular a partir dos programas, inserção dos alunos já no início do curso em cenários de prática, estímulo à interdisciplinaridade, diversificação de práticas avaliativas e uso de metodologias ativas. Na análise fatorial, verificou-se que os cursos de Medicina e Biomedicina mostram-se mais refratários às contribuições dos programas Pró-Saúde e Pet-Saúde. Outros desafios foram apontados, como carga horária excessiva, que dificulta a integração entre cursos e o desenvolvimento da competência em administração/gestão ainda incipiente. A junção dos programas na forma Propet-Saúde deve ser fortalecida, pois evidenciou grande assertividade na organização curricular, bem como na integração ensino-serviço, aspecto central para o fortalecimento do Sistema Único de Saúde (SUS). 


\section{KEY-WORDS}

- Evaluation.

- Pró-Saúde.

- PET-Saúde.

- Curricular Restructuring.

\section{ABSTRACT}

This study problematized the perception of stakeholders in the health care field at the Federal University of Goiás regarding the impact of the National Program for Professional Training and Reorientation in Health under the Program for Education for Work in Health (Propet-Saude) on curricular restructuring. This cross-sectional study included 300 students, tutors, preceptors, teachers and graduation coordinators from seven courses. A Likert-type instrument was constructed and validated with the following dimensions: D1: curricular restructuring and the principles of PropetSaúde and D2: the pedagogical project of the Propet-Saúde course. The instrument was validated considering semantic, cultural and conceptual equivalence, assignment of points (from 1 to 4), and randomization of the assertions tested. The score generated three interpretive intervals: from 1 to 1.99 (dissatisfaction); 2 to 2.99 (relative satisfaction) and 3 to 4 (satisfaction). The instrument was applied following a pre-test and the placement of the contributors. The instrument was then statistically validated for dispersion and reliability. Factorial analysis was performed considering function and course. Dispersion through linear correlation showed a loss of 4 assertions (11.77\%), signifying excellent content validation. Reliability was 0.93 ( $p<0.01)$, showing excellent statistical density. The general averages of D1 and D2 were 3.09 and 3.11 respectively, indicating satisfaction among the respondents. In conclusion, the participants recognize important advances such as curricular restructuring from these programs, placement of students already at the beginning of the course into practice scenarios, motivation toward interdisciplinarity, diversification of evaluation practices, and use of active methodologies. After factorial analysis, it was shown that medical and biomedical courses are more resistant to the contributions of the Pró-Saúde and PET-Saúde Programs and other hurdles were identified, such as excessive workload that hinders the integration of courses and the development of still-evolving administration and management skills. The interaction of the programs in Propet-Saúde should be strengthened, as it demonstrated significant assertiveness in curricular organization as well as in the teaching and service integration, a central aspect for the strengthening of Brazilian Public Health.

Recebido em: 27/11/18

Aceito em: 17/12/18

\section{INTRODUÇÃO}

As transformações estruturais, em especial políticas, econômicas, culturais sociais e tecnológicas das primeiras décadas do século XXI, têm provocado um debate aprofundado sobre a formação de profissionais da área de saúde ${ }^{1}$.

A partir da Reforma Sanitária, iniciada na década de 1970, vem-se discutindo a formação dos profissionais de saúde em nosso país. Esse movimento visou romper com práticas vigentes em diversas escolas superiores de formação em saúde, cujos projetos pedagógicos e ambientes de ensino-aprendizagem estavam conectados ao modelo biomédico flexneriano, caracterizado pela centralidade da concepção biológica da doença. Embora as mudanças pedagógicas sejam desafiadoras, vislumbra-se que a formação de profissionais deve atender às necessidades de saúde da população e ser coerentes com o modelo de atenção do Sistema Único de Saúde (SUS) ${ }^{2}$.
Uma iniciativa que sinalizou possibilidades de mudanças foi a proposição das Diretrizes Curriculares Nacionais (DCN) para os cursos da área da saúde, em 2001, em consonância com as diretrizes do SUS e com a Lei de Diretrizes e Bases da Educação, de 1996³ . Esse documento propôs novas estratégias de ensino e avaliação da aprendizagem, além de reconfigurar o espaço da formação, explicitando a necessidade de integração entre ensino e serviço para o planejamento e discussão do perfil do profissional que o País deseja formar e o seu papel na sociedade ${ }^{4}$ Em 2014, as DCN do curso de Medicina foram reestruturadas, fortalecendo o compromisso de formação profissional com foco na atenção básica e na Medicina da Família ${ }^{5}$.

Foram formuladas, ainda, outras iniciativas, referentes à implantação de políticas públicas indutoras da reorientação da formação em saúde, que visaram estimular e incentivar as mudanças curriculares, à superação do modelo hegemônico 
na formação superior na área da saúde e à articulação entre os serviços de saúde e o ensino. Entre elas, destacaram-se o Programa Nacional de Reorientação da Formação Profissional em Saúde (Pró-Saúde) ${ }^{6}$ e o Programa de Educação pelo Trabalho para a Saúde (PET-Saúde) 7 .

O Pró-Saúde buscou promover transformações nos processos de ensino e aprendizado, alicerçado em três eixos orientação teórica, cenários de prática e orientação pedagógica -, a fim de proporcionar uma formação que abarque, além da dimensão técnica, as necessidades sociais, culturais e econômicas da população ${ }^{6}$. Complementarmente, o PET-Saúde visou ao processo de integração ensino-serviço-comunidade, propiciando aos estudantes da área da saúde a iniciação ao trabalho, estágios e vivências ${ }^{7}$.

A Universidade Federal de Goiás (UFG), induzida pelos pressupostos contidos nos programas citados, buscou enfrentar o desafio de implementar a reorientação da formação de seus alunos pautada no atendimento das necessidades de saúde da população, aproximando-se dos cenários de prática ofertados pela Secretaria Municipal de Saúde de Goiânia, por meio da adesão aos cinco editais do Pró-Saúde e do PET-Saúde. Apesar disso, não foram realizadas avaliações do impacto da sua implantação sobre a reestruturação dos cursos. Assim, este estudo buscou identificar a percepção dos atores envolvidos no Pró-Saúde articulado ao PET-Saúde (Propet-Saúde) sobre a reestruturação curricular dos cursos de graduação da área da saúde da UFG.

\section{PERCURSO METODOLÓGICO}

Trata-se de um estudo transversal de abordagem quantitativa, desenvolvido em 2014, com aplicação de um instrumento atitudinal do tipo Likert aos atores envolvidos no desenvolvimento do Programa Nacional de Reorientação da Formação Profissional em Saúde (Pró-Saúde), articulado ao Programa de Educação pelo Trabalho para a Saúde - PET-Saúde (Propet-Saúde) da Universidade Federal de Goiás (UFG), instituição localizada no município de Goiânia, Goiás, Região Centro-Oeste do Brasil.

A população do estudo englobou os atores participantes e ex-participantes do Edital nº 024 de 15 de dezembro de 2011 da UFG, tais como preceptores, tutores e estudantes bolsistas. Também participaram os coordenadores dos cursos envolvidos no edital: Biomedicina, Educação Física, Enfermagem, Farmácia, Medicina e Nutrição, exceto Odontologia, pois a coordenadora era pesquisadora do presente estudo, além dos professores e estudantes não bolsistas desses cursos.

A amostra de conveniência foi constituída por 120 estudantes bolsistas, 31 estudantes ex-bolsistas, 60 preceptores, 14 ex-preceptores, nove tutores, três ex-tutores, 28 professores (quatro professores de cada curso) e a proporção de dois estudantes não bolsistas do respectivo ano de ingresso do bolsista ou ex-bolsista, sorteados aleatoriamente, totalizando 302 estudantes e os seis coordenadores de curso. Ao final, o instrumento de coleta de dados foi respondido por 321 pessoas, porém foram descartados 21 (6,5\%) por preenchimento incompleto, totalizando uma amostra de 300 participantes.

Foi aplicado o teste de Anova para as dimensões a fim de garantir que as diferenças entre os valores encontrados fossem de fato significativas. Considerou-se um nível de significância $(\alpha)$ de $5 \%$; o nível de significância é a probabilidade de se cometer um erro do tipo I, ou seja, rejeitar a hipótese nula quando esta é verdadeira. Por meio do p-valor do teste, constatamos que a hipótese foi satisfeita no nível de $5 \%$ de significância $(\chi \chi 2(2)=3,465, p p=0,177)$, sem a necessidade de utilização de correções ${ }^{9-11}$.

A opção pela escala Likert se deu por esta ser considerada uma potente ferramenta para um aprofundamento em estudos avaliativos ${ }^{8-11}$. A escala caracteriza-se por conter assertivas positivas e negativas sobre o objeto estudado, e nela o respondente apresenta sua percepção sobre o objeto pesquisado por meio de sua concordância ou discordância da assertiva.

O instrumento de coleta de dados foi construído coletivamente numa oficina com representantes de todos os atores envolvidos, tais como docentes, tutores, preceptores e estudantes, à luz dos documentos citados. O instrumento foi composto por 34 assertivas (A), divididas em quatro dimensões: D1) reestruturação curricular e os princípios do Propet-Saúde; D2) projeto pedagógico do curso e os princípios do Propet-Saúde; D3) impacto dos cenários de práticas na integração ensino-serviço; e D4) contribuição do programa Propet-Saúde para a formação interprofissional. No presente trabalho, serão analisadas as dimensões D1 e D2.

Na construção da D1, buscou-se identificar questões como a presença da interdisciplinaridade e da integralidade como eixos da formação em saúde; adoção de metodologias ativas no processo de ensino-aprendizagem; presença de atividades integradoras; fortalecimento da atenção básica e promoção da saúde; realização de avaliação formativa e equilíbrio entre a formação técnica, social e ética. A D2 propôs-se analisar o trabalho em equipe e as competências definidas nas DCN, a integração entre o ciclo básico e profissional, além do fortalecimento do SUS. No Quadro 1 encontram-se as duas dimensões da escala Likert e suas respectivas assertivas.

O instrumento foi validado em seu conteúdo observando-se a equivalência semântica (significado das palavras), a cultural (termos e situações cotidianos diferentes entre as cul- 


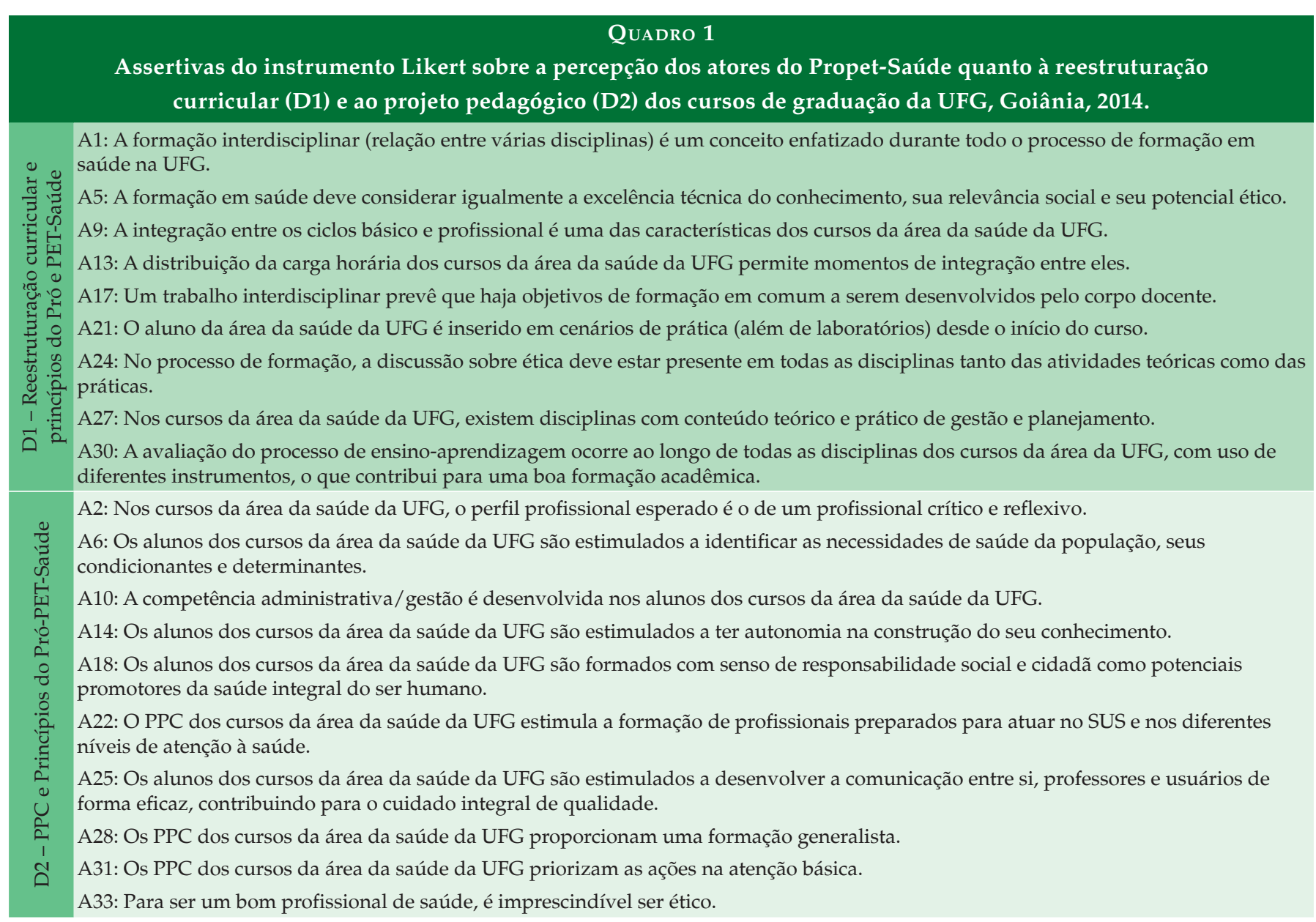

turas) e a conceitual (palavras que possuem significados culturais diferentes). Em seguida, as assertivas foram pontuadas em escalas que variaram de $1 / 2 / 3 / 4$ a $4 / 3 / 2 / 1$, associadas a uma escala atitudinal de concordância plena $(\mathrm{CP})$ a discordância plena (DP), com os seguintes termos intermediários: inclinado a concordar (IC) e inclinado a discordar (ID). Em seguida, as assertivas foram randomizadas para a aplicação do instrumento.

Após a aplicação do instrumento, as questões foram validadas estatisticamente quanto a sua dispersão e confiabilidade. Em relação à dispersão, foi aplicado o coeficiente de correlação linear ${ }^{8,12}$. O coeficiente de correção linear (r) foi calculado para todas as asserções na simulação da primeira administração, visando a sua depuração com a eliminação das asserções com correlação linear inferior a 0,30. Em seguida, o valor de (r) foi calculado novamente, numa segunda simulação, considerando apenas as asserções validadas em todos os instrumentos respondidos e, novamente, houve a eliminação das asserções com correlação linear inferior a 0,20.
A literatura admite perdas de até $40 \%$ quanto à dispersão. Nesta pesquisa, obteve-se 11,77\%, o que evidencia ótima validação de conteúdo. Assim, foram excluídas duas assertivas, sendo uma de cada dimensão (D1, A5 e D2, A33). Foram elas: "A formação em saúde deve considerar igualmente a excelência técnica do conhecimento, sua relevância social e seu potencial ético" (média 3,72) e "Para ser um bom profissional de saúde é imprescindível ser ético" (média 3,91). Como se trata de dois consensos positivos, não houve dispersão, daí a exclusão. Contudo, esses dados devem ser analisados qualitativamente, pois indicam uma compreensão extremamente adequada quanto aos princípios contemporâneos da formação em saúde, bem como a centralidade da ética nesse campo.

Em seguida, procedeu-se ao coeficiente de confiabilidade pelo método de teste-reteste ${ }^{13}$. Neste estudo, foi utilizado o método de split-half, conforme descrito por Ritz ${ }^{14}$, com o critério considerado de aceitação de um mínimo de $80 \%$, equivalendo a $R \geq 0,80$. Nesta investigação, obteve-se $0,93 \%$, o que denota excelente sustentação dos dados encontrados. 
A interpretação das percepções baseou-se na pontuação média alcançada após o tratamento estatístico, sendo que de 1 a 1,99 pontos a percepção foi considerada insatisfatória, mostrando a necessidade de uma intervenção em curto prazo; de 2 a 2,99 pontos (relativa satisfação), a percepção é reveladora de aspectos a serem melhorados, exigindo medidas de médio prazo; e de 3 a 4 pontos (satisfação), a percepção é positiva e pode ser potencializada.

Este estudo foi aprovado pelo Comitê de Ética em Pesquisa da Universidade Federal de Goiás, sob o parecer $\mathrm{n}^{\circ}$ $571.173 / 2014$.

\section{RESULTADOS E DISCUSSÃO}

A população da pesquisa foi composta pelos representantes dos cursos integrantes do edital que instituiu o Programa Nacional de Reorientação da Formação Profissional em Saúde (Pró-Saúde), articulado ao Programa de Educação pelo Trabalho para a Saúde - PET-Saúde (Propet-Saúde). Assim, participaram estudantes, professores, tutores e preceptores dos serviços de saúde, ou seja, a comunidade acadêmica, exceto os servidores técnico-administrativos Os dois eixos de análise adotados para este estudo foram função exercida e categoria profissional Tabela 1.

\begin{tabular}{|c|c|c|c|c|c|}
\hline \multicolumn{6}{|c|}{$\begin{array}{l}\text { TABELA } 1 \\
\text { Eixos de análise da pesquisa quanto à percepção dos } \\
\text { participantes do Propet-Saúde sobre a reestruturação } \\
\text { curricular dos cursos de graduação da UFG, Goiânia, } 2014 .\end{array}$} \\
\hline \multicolumn{6}{|c|}{ Eixos de análise } \\
\hline Função & n & $\%$ & $\begin{array}{c}\text { Categoria } \\
\text { profissional }\end{array}$ & n & $\%$ \\
\hline Coordenadores & 03 & 1,00 & Biomedicina & 17 & 5,67 \\
\hline Professores & 15 & 5,00 & Educação Física & 31 & 10,33 \\
\hline Tutores & 08 & 2,67 & Enfermagem & 63 & 21,00 \\
\hline Ex-tutores & 03 & 1,00 & Farmácia & 38 & 12,67 \\
\hline Preceptores & 38 & 12,67 & Medicina & 37 & 12,33 \\
\hline Ex-preceptores & 02 & 0,67 & Nutrição & 52 & 17,33 \\
\hline Estudantes bolsistas & 69 & 23,00 & Odontologia & 62 & 20,67 \\
\hline Estudantes ex-bolsistas & 11 & 3,66 & & & \\
\hline Estudantes não bolsistas & 151 & 50,33 & & & \\
\hline Total & 300 & & Total & 300 & 100,00 \\
\hline
\end{tabular}

A análise fatorial considerou dois parâmetros: os cursos da UFG e as funções exercidas pelos respondentes. Em ambas as dimensões, os cursos de Biomedicina, Medicina, estudantes ex-bolsistas, tutores e coordenadores de curso apontaram a necessidade de melhorias em médio prazo. Para o curso de Enfermagem e ex-tutores, essa mesma resposta foi apenas para a Dimensão 1 (reestruturação curricular e princípios do Propet). Os demais cursos e funções demonstraram percep- ção satisfatória da influência do Propet-Saúde no âmbito desta instituição de ensino superior (Gráfico 1).

O Gráfico 2 mostra as percepções obtidas na dimensão D1 $(3,09)$, na qual os respondentes demonstraram relativa satisfação com aspectos como melhor distribuição da carga horária, o que permite a presença de momentos de integração entre os cursos (A 13), maior conteúdo relacionado à gestão e planejamento (A 27) e, por último, o aperfeiçoamento relativo à integração dos ciclos básico e profissional (A9), respostas que sinalizam a necessidade de mudanças a médio prazo.
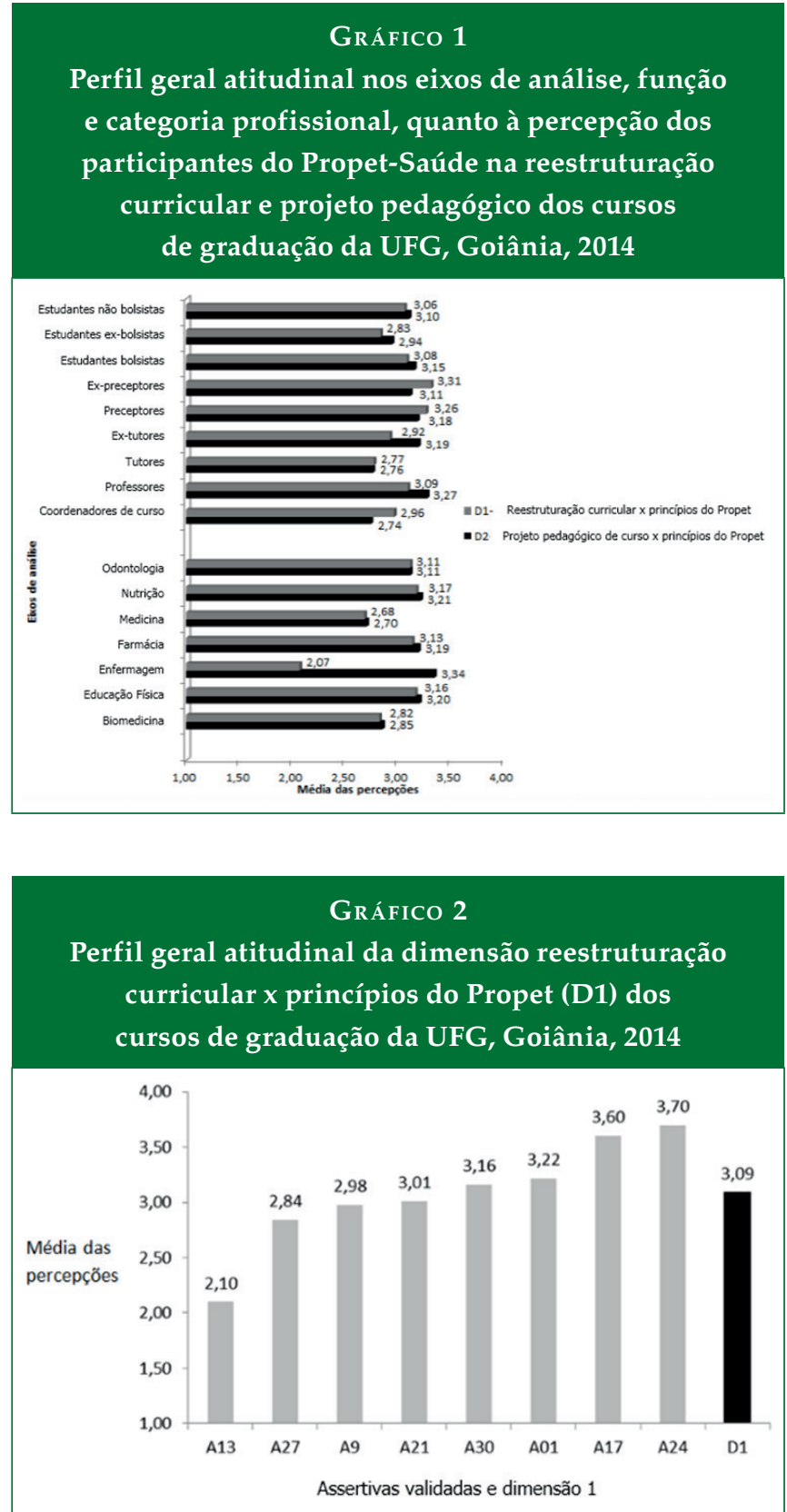
A integração entre os estudantes de diferentes cursos tem sido discutida frequentemente nos planejamentos pedagógicos que ocorrem semestralmente na UFG. Entretanto, os horários não flexíveis em que ocorrem as disciplinas, além do número máximo de estudantes aceitos nas Unidades Básicas de Saúde, têm dificultado os momentos de integração. Sabe-se que atividades e vivências multidisciplinares levam à aprendizagem de conteúdos não abordados nos cursos de graduação, mas são significativos para a prática profissional ${ }^{15}$. Portanto, buscar formas de instituir, nos cursos pesquisados, espaços de integração e atuação multiprofissional representaria um avanço na reestruturação curricular.

Também foram evidenciados nesta dimensão aspectos a melhorar, como o desenvolvimento da competência de gestão e planejamento dos estudantes da área da saúde ${ }^{16}$, como requerido pelas $\mathrm{DCN}^{3,17}$. Para isso, a diversificação dos cenários de prática, que é um dos princípios do Propet-Saúde, é fundamental para possibilitar a construção de conhecimento. Percebe-se, assim, a necessidade de institucionalizar e aprimorar a relação entre as universidades e os serviços de saúde. Nesse sentido, na discussão da implantação do Contrato Organizativo de Ação Pública Ensino-Saúde (Coapes), que ocorreu entre a Secretaria Municipal de Goiânia e as instituições de ensino superior, foram utilizados os cenários de prática dessa Secretaria, uma estratégia que proporcionou melhoria das condições de inserção dos estudantes nos serviços de saúde.

Outro aspecto apontado foi o crônico problema das organizações curriculares em saúde que é a falta de integração entre os ciclos básico e profissional. Isto reflete, em parte, a presença da dicotomia idealizada por Flexner em 1910, em que o estudante fazia a aprendizagem das matérias básicas em laboratórios e depois aprendia a tratar a doença no hospital, longe do contexto social, coletivo e da comunidade que a produziu ${ }^{18}$, o que contraria as DCN e os avanços do conhecimento, principalmente na área da promoção da saúde. Os programas de reorientação da formação preconizaram melhor relação entre os ciclos básico e profissional por meio de uma integração curricular horizontal que favoreça a integração de conhecimentos complexos e complementares ${ }^{7}$.

Merece destaque a percepção positiva dos respondentes quanto a aspectos importantes da reestruturação curricular pautada pelos princípios do Propet-Saúde, como a inserção do estudante em cenários de prática desde o início do curso, a ênfase no conceito de formação interdisciplinar com objetivos comuns junto ao corpo docente, a discussão sobre ética nas atividades teóricas e práticas e o uso de diferentes instrumentos para avaliar o processo ensino-aprendizagem.
A participação da UFG nos programas de reorientação da formação propiciou a inserção dos estudantes nos cenários de prática desde o início dos cursos. Isto foi estimulado por meio de vagas para bolsistas desde o primeiro semestre do curso nos editais PET-Saúde e pela elaboração dos novos projetos pedagógicos para os cursos de Farmácia, Medicina, Educação Física e Nutrição, que oferecem no primeiro e segundo período disciplinas com atividades práticas na atenção básica de saúde. Ao adaptarem os conteúdos à prática da atenção básica e inserirem os estudantes de forma precoce nessas práticas, os cursos favoreceram as iniciativas para a futura fixação dos profissionais de saúde em locais remotos ${ }^{19}$.

O Gráfico 3 apresenta a percepção dos respondentes sobre a dimensão D2 - "Projeto Pedagógico de Curso e Princípios do Propet dos Cursos de Graduação da UFG". Apesar das iniciativas para o início precoce das práticas na atenção básica de saúde, percebe-se que essas atividades ainda são relativamente priorizadas nos projetos pedagógicos dos cursos. Ainda assim, os atores concordaram quanto à presença da formação de profissionais para atuação no SUS em diferentes níveis de atenção à saúde com a identificação das necessidades de saúde, seus condicionantes e determinantes. Reconheceram o estímulo à autonomia dos estudantes para construção do conhecimento, bem como o desenvolvimento da comunicação. Identificaram também a presença de uma formação generalista, com senso de responsabilidade social, cidadã e promotora da saúde, que os instiga a se tornarem profissionais crítico-reflexivos.

GrÁFICO 3

Perfil geral atitudinal da dimensão projeto

pedagógico de curso $x$ princípios do Propet (D2)

dos cursos de graduação da UFG, Goiânia, 2014

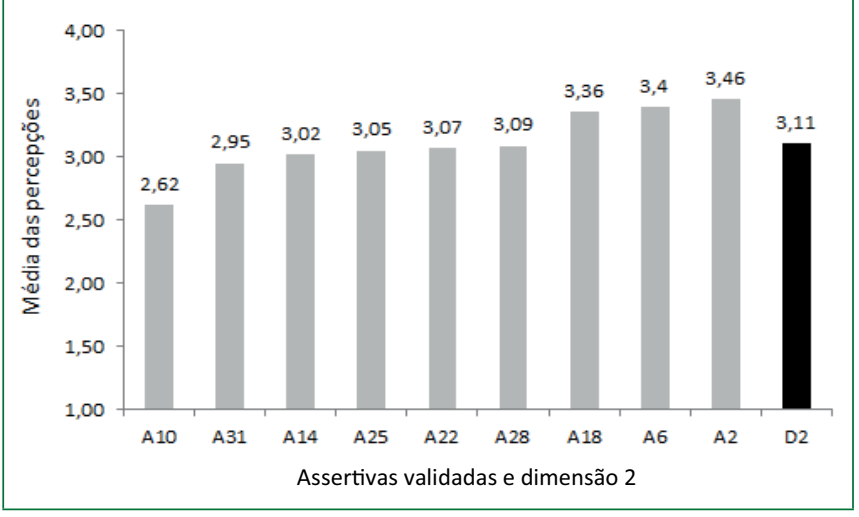

Destaca-se a percepção satisfatória quanto ao uso de diferentes instrumentos para avaliação do processo ensino-aprendizagem, por constituírem um resultado importante, oriundo 
da formação docente proporcionada por meio das oficinas e cursos realizados com os recursos advindos do Pró-Saúde/ UFG, como os de metodologias ativas e avaliação da aprendizagem.

Este estudo possibilitou conhecer avanços e desafios na formação em saúde na ótica de estudantes, preceptores e professores de cursos participantes de programas de reorientação da formação numa universidade pública. O Propet-Saúde impulsionou avanços na relação ensino-serviço, na reestruturação curricular e no projeto pedagógico dos cursos. Este estudo também possibilitou perceber nesses avanços a necessidade da integração entre os ciclos básico e profissional, de momentos de integração a partir de melhor distribuição de carga horária nos cursos, de conteúdos de gestão, planejamento e administração nos cursos e da priorização das ações de ensino na atenção básica.

Sugere-se que as instituições de ensino superior reflitam sobre a necessidade de uma política institucional de valorização das atividades de desenvolvimento dos docentes da área da saúde $\mathrm{e}^{20,21}$, principalmente quanto à discussão dos objetivos de aprendizagem e às estratégias metodológicas para a integração dos ciclos básico e profissional, considerando a atenção básica de saúde como um cenário de prática prioritário, a fim de proporcionar aos discentes a formação preconizada pelas DCN.

\section{CONSIDERAÇÕES FINAIS}

Esta pesquisa evidenciou a assertividade dos princípios propostos pelo Propet-Saúde e como eles têm embasado a reestruturação curricular dos cursos pesquisados na UFG. No entanto, percebem-se, ainda, níveis distintos de avanços, e esta constatação demonstra a necessidade de intervenções em aspectos relevantes, como integração multiprofissional, gestão/ administração, integração básico-profissional e priorização de atividades na atenção básica de saúde em curto/médio prazo, a fim de implementar ajustes para uma formação alinhada às necessidades da população e voltada ao modelo de atenção do SUS.

A formação em saúde demonstrou seu caráter multifatorial, apontando aspectos de infraestrutura, respeito ao tempo de mudança, atores estimulados para avanços e apropriação das políticas existentes com tradução nos currículos vigentes.

É fundamental ter a noção precisa de processo e compromisso com a formação acadêmica, para que se formem profissionais de saúde capazes de contribuir, de fato, para a superação dos desafios vigentes num país ainda tão desigual.

Espera-se que os programas Pró-Saúde e PET-Saúde sejam assumidos pelo próximo governo como fundamentais para avançarmos na formação em saúde no Brasil e que eles sejam dispostos como políticas públicas e não mais como programas, para que se possa, cada vez mais, atingir na totalidade a formação estabelecida nas DCN.

\section{REFERÊNCIAS}

1. Furlanetto DLC, Lima AAL, Silva Júnior JW, Bastos MM, Pinho DLM. Avaliação dos estudantes do Pró-PET - Saúde sobre as contribuições do Programa Pró-Saúde nos cursos de saúde Campus Darcy Ribeiro - Universidade de Brasília. Actas Saúde coletiva. 2015; 9(1):11-24.

2. Moraes BA,Costa NMSC. A formação em saúde no Brasil: o currículo e as políticas indutoras de reorientação da formação. In: Costa NMSC, Pereira ERS. Ensino na Saúde: transformando práticas profissionais. Goiânia: Gráfica UFG; 2015. 17-34.

3. Brasil. Parecer n⿳0 1.133 de 7 de outubro de 2001. Dispõe as Diretrizes Curriculares para os cursos de graduação de Enfermagem, Farmácia, Medicina, Nutrição e Odontologia. Brasília: Ministério da Saúde/Educação; 2001.

4. Soares NT, Aguiar AC. Diretrizes curriculares nacionais para os cursos de nutrição: avanços, lacunas, ambiguidades e perspectivas. Rev. Nutrição. 2010; 23(5): 895-905

5. Brasil. Portaria interministerial $\mathrm{n}^{\mathrm{o}} 116$, de 3 de abril de 2014. Dispõe as Diretrizes Curriculares para o curso de graduação de Medicina. Brasília: Ministério da educação/ saúde; 2014.

6. Brasil. Ministério da Saúde. Ministério da Educação. Portaria interministerial n. 2.101, de 3 de novembro de 2005. Institui o Programa Nacional de Reorientação da Formação Profissional em Saúde - Pró-Saúde - para os cursos de Graduação em Medicina, Enfermagem e Odontologia. Diário Oficial da União: Brasília; 2005.

7. Brasil. Ministério da Saúde. Portaria Interministerial $\mathrm{n}^{\mathrm{o}}$ 1.802 de 26 de agosto de 2008.Institui o Programa de Educação pelo Trabalho para a Saúde - PET - Saúde. Diário Oficial da União: Brasília; 2008.

8. Ferreira BJ. Inovações na Formação Médica: Reflexos na Organização do Trabalho Pedagógico.Tese de Doutorado. Faculdade de Educação da Unicamp; 2004.

9. Calvano S, Fereira BJ, Domene SMA.Avaliação da usabilidade do Guia Alimentar Digital móvel segundo a percepção dos usuários. Ciênc. saúde coletiva. 201419(5): 1437-1446.

10. Cardoso RB, JansenB. Programa de Educação Permanente para o Uso do Prontuário Eletrônico do Paciente na Enfermagem. Journal of health informatics. 2017; 9(1): 25-35.

11. Cardoso RB, Paludeto SB, Ferreira BJ. Programa de educação continuada voltado ao uso de tecnologias em saúde: 
percepção dos profissionais de saúde. Rev. Bras. Ciên. Saúde v. 22, n³ João Pessoa, Paraíba, 2018.

12. Moraes SG, Justino ML, Jansen BF, Barbosa EP, Bruno LFC, Pereira LAV. Development and validation of strategy to assess teacching methods in undergraduate disciplines. In: Progress in Education. 2012. Volume 28. Nova Science Publishers.

13. Schimidt, MJ. Understanding and Using Statistics Basic Concepts. Massachusetts, USA: D.C. Health and Company. 1975.

14. Ritz MC. Qualidade de Vida no Trabalho: Construindo, Medindo e Validando uma Pesquisa. Instituto de Matemática, Estatística e Computação Cientifica (IMECC), UNICAMP, Campinas; 2000.

15. Leal JAL, Melo CMM, Veloso RBP, Juliano IA. Novos espaços de reorientação para formação em saúde: vivências de estudantes. Interface. 2015; 19(53): 361-71.

16. Fadel CB, Baldani MH. Percepções de formandos do curso de odontologia sobre as Diretrizes Curriculares Nacionais. Trab. Educ. Saúde. 2013; 11(2): 339-354.

17. Dias HS, Lima LD, Teixeira MA. A trajetória da política nacional de reorientação da formação profissional em saúde no SUS. Ciênc. saúde coletiva. 2013; 18(6): 1613-1624.

18. Pagliosa FL, Da Ros MA. O Relatório Flexner: Para o Bem e Para o Mal. Rev. bras. educ. med. 2008; 32(4): 492-499.

19. Scheffer M. Programa Mais Médicos: em busca de respostas satisfatórias. Interface. 2015; 19(54): 637-640.

20. Cardoso CGLV, Costa NMSC,Moraes BA. Desafios da formação pedagógica em nutrição. Cienc.docencia y tecnologia. 2016; 27(53): 33-49.

21. Sobral DJ.. Três Casos de Inovação Curricular no Panorama recente (1964-1988) da Educação médica Brasileira: Subsídios de um retrospecto Baseado na revisão de documentos. Rev. Bras. Educ. Med. 2014; 38(4), 493-501.

\section{INFORMAÇÕES SOBRE O ESTUDO}

Esta pesquisa obteve financiamento do Conselho Nacional de Desenvolvimento Científico e Tecnológico, edital MCTI/ CNPq/MS - SCTIE - Decit nº 08/2013, e foi aprovada pelo Comitê de Ética em Pesquisa da Universidade Federal de Goiás (CEP/UFG) sob o protocolo n 571.173/2014.

\section{CONTRIBUIÇÃO DOS AUTORES}

Bibiana Arantes Moraes, Lucilene Maria de Sousa, Ida Helena Carvalho Francescantonio Menezes, Maria Goretti Queiroz e Nilce Maria da Silva Campos Costa participaram da concepção, delineamento, interpretação dos dados e de todas as fases de escrita do artigo. Marília Mendonça Guimarães participou da interpretação dos dados, redação do artigo e revisão final. Beatriz Jansen Ferreira participou da análise, interpretação dos dados, redação e revisão crítica do artigo.

\section{CONFLITO DE INTERESSES}

Os autores declararam não existirem qualquer tipo de conflito de interesses.

\section{ENDEREÇO PARA CORRESPONDÊNCIA}

1ª Avenida, s/n, Setor Universitário, CEP 74605-020, Goiânia, Goiás, Brasil.

bibiananutri20@gmail.com 